\title{
A novel pre-operative model to predict 90-day surgical mortality in patients considered for renal cell carcinoma surgery
}

Adam C. Calaway*, M. Francesca Monn, Clinton D. Bahler, Clint Cary, and Ronald S. Boris *Corresponding Author

Adam C Calaway*

535 N Barnhill Dr. Suite 150

Indianapolis, IN 46202

Phone: $317-278-4881$

Fax: 317-278-0499

e-mail: calawaya@iupui.edu

Affiliation: Indiana University School of Medicine, Department of Urology

M. Francesca Monn

e-mail: mmonn@iupui.edu

Affiliation: Indiana University School of Medicine, Department of Urology

Clinton D. Bahler

e-mail: cdbahler@iupui.edu

Affiliation: Indiana University School of Medicine, Department of Urology

Clint Cary

e-mail:kcary@iupui.edu

Affiliation: Indiana University School of Medicine, Department of Urology

Ronald S. Boris

e-mail: rboris@iupui.edu

Affiliation: Indiana University School of Medicine, Department of Urology

Running head: Predicting 90-day surgical mortality for RCC

Abstract Word Count: 250

Manuscript Word Count: 2,750

\section{Tables: 4}

Figures: 4

Funding: This research did not receive any specific grant from funding agencies in the public, commercial, or not-for-profit sectors.

This is the author's manuscript of the article published in final edited form as:

Calaway, A. C., Monn, M. F., Bahler, C. D., Cary, C., \& Boris, R. S. (2018). A novel preoperative model to predict 90-day surgical mortality in patients considered for renal cell carcinoma surgery. Urologic Oncology: Seminars and Original Investigations, 36(10), 470.e11-470.e17. https://doi.org/10.1016/j.urolonc.2018.07.006 


\begin{abstract}
:
Introduction: Surgical benefits for renal cell carcinoma must be weighed against competing causes of mortality, especially in the elderly patient population. We used a large cancer registry to evaluate the impact of patient and cancer specific factors on 90-day mortality (90DM). A nomogram to predict the odds of short-term mortality was created.
\end{abstract}

Materials and Methods: The National Cancer Database was queried to identify all patients with clinically localized, non-metastatic disease treated with partial or radical nephrectomy. Using a random sample of $60 \%$, multiple logistic regression with 90DM outcomes was performed to identify pre-operative variables associated with mortality. Variables included age, sex, race, comorbidity score, tumor size, and presence of a thrombus. A nomogram was created and tested on the remaining $40 \%$ of patients to predict 90DM.

Results: 183,407 patients met inclusion criteria. Overall 90DM for the cohort was 1.9\%. All preoperative variables significantly influenced the risk of 90DM. Patient age was by far the strongest predictor. Nomogram scores ranged from 0-12. Compared to patients with 0-1 points, those with 2-3 (OR 2.89, 2.42-3.46; $\mathrm{p}<0.001)$, 4-5 (OR 6.25, 5.26-7.43; $\mathrm{p}<0.001)$, and $>6$ (OR $12.86,10.83-15.27 ; \mathrm{p}<0.001)$ were at incrementally significantly higher odds of 90DM. Being $>80$ years of age alone placed patients into the highest risk of surgical mortality.

Conclusions: Management of localized kidney cancer must consider competing causes of mortality, especially in elderly patients with multiple co-morbidities. We present a preoperative tool to calculate risk of surgical short-term mortality to aid surgeon-patient counseling.

Key Words: Renal Cell Carcinoma, Peri-Operative Mortality, Risk Prediction, Patient Counseling, Nomogram 


\section{Introduction:}

Kidney cancer is the $8^{\text {th }}$ leading cancer diagnosis in the United States with an estimated 63,990 of new cases resulting in 14,400 deaths based on 2016 data (1). In contrast to many of the other most commonly diagnosed malignancies, the incidence of kidney cancer has risen over the last few decades in men and women of every racial and ethnic group (2). The rise in incidence of kidney cancer has coincided with a downward drift stage migration likely secondary to the more frequent diagnosis of small, incidental renal masses on cross-sectional imaging (3-6). This rise in incidence, however, has not been observed in conjunction with a decrease in mortality despite increases in surgical treatment (5). In fact, in some studies, mortality from renal cell cancer has risen calling into question current treatment paradigms thus stressing appropriate patient selection for operative procedures $(2,5,7)$.

Surgical resection remains the gold standard therapy for patients with renal cell carcinoma. The survival benefit from surgery for renal cell carcinoma is variable and is dependent upon patient and disease specific factors. Age and associated medical comorbidities weigh heavily on patient's overall survival and short term surgical morbidity and mortality $(8,9)$. Tumor specific characteristics such as tumor grade, stage and local/metastatic extent confer different risks of morbidity and mortality (10). Previously, various studies have evaluated predictors of shortterm surgical morbidity, mortality and long-term overall-survival (11-13). These studies have been influential in pre-operative patient counseling.

In our current study, we sought to expand on these previous studies to evaluate pre-operative patient and tumor specific variables to evaluate their impact on short-term surgical mortality. We hypothesized that age, tumor size and stage, gender, race and co-morbidity status affect 
short-term surgical mortality. Using the National Cancer Database (NCDB), we evaluated the impact of these variables on 90-day mortality to develop a nomogram to predict the odds of short-term surgical mortality to help aid in patient counseling.

\section{Materials and Methods:}

\section{1}

Data Source:

The NCDB is a joint cancer registry that was established in 1989 by the Commission on Cancer of the American College of Surgeons and the American Cancer Society. The database serves as a comprehensive clinical surveillance resource for cancer care in the United States $(14,15)$. The NCDB captures $>70 \%$ of all newly diagnosed cancer cases from over $>1,500$ Commission on Cancer-accredited cancer programs in the United States and Puerto Rico.

\section{2}

\section{Study Population:}

The NCDB user file for renal cell carcinoma was queried from 2003-2011. The American Joint Committee on Cancer staging system was used to identify patients with clinically localized, nonmetastatic renal cell carcinoma (cT1-4, N0, M0). Patient's with clinically suspected or histologically confirmed locally advanced or metastatic lesions (N+: 22,956; $\geq \mathrm{M} 1: 40,580)$, upper-tract urothelial cell carcinoma $(\mathrm{n}=34,279)$ and who were not treated surgically were excluded $(\mathrm{n}=23,481)$. Patients with incomplete Charlson-Deyo co-morbidity data $(\mathrm{n}=68,324)$, pathologic surgical staging $(\mathrm{n}=67,414)$, or 90 -day mortality data $(\mathbf{9 0 D M})$ were excluded $(\mathrm{n}=35,446)$. Ultimately, 183,407 met inclusion criteria.

\section{3}

Co-variables and Endpoints: 
90-day mortality was used as the primary endpoint for analysis. Co-variables used to construct the multivariable model included pre-operative patient (age, gender, race and Charlson-Deyo comorbidity scores) and tumor specific variables (size and presence or absence of a thrombus) that were presumed known to the surgeon prior to the operation. Tumor size was used as a surrogate for tumor stage. Since the NCDB incompletely captures radiographic involvement of the inferior vena cava (IVC) in clinical stage variables, we elected to use pathologic demonstration of an IVC thrombus as a reasonable presumption that its involvement would be known preoperatively in all cases with rare exceptions. Finally, an exploratory analysis excluding patients with pT1 disease was done to identify pre-operative predictors of 90DM in patients who would likely not be candidates for active surveillance.

\section{4}

Statistical Analysis:

Descriptive analysis of the data was performed using Pearson's chi-squared test for categorical data, Student's $t$-test for continuous data, and the Mann-Whitney test in the case of non-normally distributed continuous data. Using a random sample of $60 \%$ of the patients in the dataset, multivariable logistic regression with the outcome of 90DM was performed to identify preoperative variables associated with mortality. Variables were determined a priori to be included in the model. A nomogram was derived from the odds ratios in the logistic regression model and tested on the remaining $40 \%$ of patients in the dataset for ability to predict 90DM. Area under the curve (AUC) was calculated for the original multivariable logistic regression model and for the tested model to confirm accuracy between the models. All statistical calculations were completed in STATA 12.1 (STATA Corp. LP, College Station, TX).

\section{Results:}


183,407 patients with clinically localized renal cell carcinoma treated with either partial or radical nephrectomy met inclusion criteria. The median age was 62 (IQR: 52-70). Most of the patients were male $(112,597,61.4 \%)$ and white $(155,771,84.9 \%)$. Most patients were classified as Charlson-Deyo co-morbidity index of $0(70.6 \%)$ with few having extensive comorbidities (Charlson-Deyo index 2, 7.3\%). The distribution of tumor stage, as defined by TNM staging, was as expected with lower stage tumors being more common (I: 69\%; II: 12\%; III: 18\%, IV: 1\%). Most patients had low-grade tumors (I-II: 59.1\%). Despite the predominance of stage I and low-grade tumors, only $29.2 \%$ of patients underwent partial nephrectomy. Overall, the 30 $(30 \mathrm{DM})$ and 90 -day mortality rates for the entire cohort were low at $0.9 \%$ and $1.9 \%$, respectively. These results and other baseline demographic, tumor specific and short-term mortality data separated by age (decade) of surgery can be visualized in Table 1 .

After separating patients into age category by decade, several findings were noted. Overall, $18.6 \%, 25.4 \%, 28.6 \%, 20.6 \%$ and $6.8 \%$ of patients were aged $\leq 49,50-59,60-69.70-79$, and $\geq 80$, respectively. Predictably, increasing age was associated with increasing comorbidity scores. Twenty five percent and $9.3 \%$ of the oldest group of patients had Charlson-Deyo index scores of 1 and 2 , respectively, compared to only $14 \%$ and $3.9 \%$ of those aged $\leq 49$ years $(p<0.001 ; p<$ 0.001). There was observed grade and stage migration as older patients were more likely to have higher grade and higher stage tumors compared to their younger counterparts (Figure 1 and 2). Additionally, surgical technique varied amongst age categories. Octogenarians were more likely to be treated with radical nephrectomy compared to the younger groups $(83.9 \%$ vs. $63.4-74.6 \%$, $\mathrm{p}<0.001$ ). In terms of short-term surgical mortality, older patients were more likely to experience 30DM and 90DM when compared to younger patients. The risk of mortality was 
highest in those aged $\geq 80$ years with 30 -day and 90 -day morality rates of $2.8 \%$ and $5.7 \%$, respectively (Table 1). 90-day mortality rates amongst the different age groups can be seen in Figure 3.

\section{1}

Development of a Nomogram:

A multivariable logistic regression model was built around pre-operative variables using our primary endpoint of 90DM in a random $60 \%$ sample of our study population. All pre-operative variables included were independent predictors of 90DM (Table 2). Older patients had higher odds of dying during the post-operative period than younger patients ( $\geq 80$ years old vs. $\leq 49$, OR 7.53 95\% CI 6.19-9.16) as did patients with more co-morbid conditions (Charlson-Deyo 2 vs. 0 , OR 2.37 95\% CI 2.08-2.69). Tumor size ( $\geq 10 \mathrm{~cm}$ vs $<4 \mathrm{~cm}$, OR $4.0695 \%$ CI 3.56-4.63) and presence or absence of a thrombus (Presence vs Absence, OR 1.90, 95\% CI 1.66-2.17) were significant predictors of 90DM. The AUC for the multivariable logistic regression model was 0.7475 .

A nomogram score was given to each pre-operative variable based on the odds ratio from the regression analysis. The highest score possible was 14 . The median (IQR) nomogram score was 1 (IQR: 0-2). Twelve percent of patients had nomogram score of 6 or higher. Nomogram groups were then made based on patient distribution (Table 3). Figure 4 graphically depicts the multivariable effect of each variable on the risk of 90-day mortality. This depicts the relative importance of age when compared to other pre-operative variables. Age $>80$ pre-operatively was the biggest predictor of 90DM. Octogenarians, based on age alone, were included in the highest nomogram group (nomogram score $\geq 6$ ). 
Testing of the Nomogram:

Multivariable logistic regression analysis was run on the remaining $40 \%$ of the study population to confirm appropriate modeling and test internal validation. In the $60 \%$ nomogram generation cohort, patients with 0-1 nomogram points compared with those with 2-3 (OR 2.89, 2.42-3.46; $\mathrm{p}$ $<0.001)$, 4-5 (OR 6.25, 5.26-7.43; $\mathrm{p}<0.001)$, and >6 (OR 12.86, 10.83-15.27; $\mathrm{p}<0.001)$ were at incrementally significantly higher odds of 90DM. The AUC for the generation cohort model was 0.7211 . The associations were confirmed in the $40 \%$ validation cohort. In this group, compared to patients with 0-1 points, those with 2-3 (OR 3.49, 2.80-4.35; p < 0.001), 4-5 (OR $6.82,5.49-8.48 ; \mathrm{p}<0.001)$ and $>6(\mathrm{OR} 14.57,11.75-18.08 ; \mathrm{p},<0.001)$ were at incrementally significantly higher odds of 90DM (Table 3). The AUC for the validation model based on the $40 \%$ was 0.7221 .

\section{3}

\section{Risk Factors of 90-Day Mortality in patients with pT2-pT4 tumors}

An exploratory multivariate model excluding patients with pT1 tumors was built around our predefined pre-operative variables. This was done to investigate if age was still an important predictor in 90DM in patients who would likely not be candidates for active surveillance. Once again, age was the greatest predictor of 90DM (Table 5).

\section{Discussion:}

Our research highlights trends in disease presentation, management and outcomes in patients with renal cell carcinoma who undergo surgical treatment. Using a prospectively maintained national cancer registry, a large patient population $(183,407$ patients) was analyzed to make generalizable conclusions. Increasing patient age corresponds with increasing tumor grade and 
stage. Correspondingly, increasing age was associated with the presence of more medical comorbidities. Elderly patients pose a difficult treatment dilemma given their increased risk of high grade and stage tumors and their competing risks of medical co-morbidities, important factors when optimizing management for patients who present with renal tumors $(8,10)$. This highlights the importance of appropriate patient selection and patient counseling, especially in the elderly when considering surgical candidacy. We evaluated 90-day surgical mortality in patients undergoing partial or radical nephrectomy to evaluate predictors of short-term mortality. Compared to younger patients $(<50)$, patients of advanced age were more likely to expire within the 30 and 90 days post-operatively. Patient age was the most important predictor of perioperative mortality which far exceeded the influence of medical co-morbidities, tumor size or advanced clinical stage. We believe that these findings highlight that patient age is a more important predictor of short-term mortality than tumor biology. Using our data, we created a nomogram to help with pre-operative decision-making and patient counseling for all patients undergoing extirpative surgery for renal cell carcinoma with the goals of surgical cure.

Previously, researchers used data from the National Cancer Institute Surveillance, Epidemiology and End Results (SEER) database to evaluate age and stage specific thirty-day mortality rates (TDM) after partial or radical nephrectomy. In a cohort of 24,535 patients, TDM increased with patient age and stage and these two factors achieved independent predictor status on logistic regression analysis (12). A second study evaluated TDM rates in patients undergoing nephrectomy in Great Britain. In analysis of 21,380 procedures performed over a three-year period, increases in TDM were associated with increasing age, stage, estimated blood loss, operative time and performance status. By including all patients treated surgically, patients with 
localized disease and metastatic disease were compared and, as expected, those with localized disease had lower odds of TDM than those undergoing cytoreductive nephrectomy (OR 0.31 95\% CI 0.18-0.54) (11). These studies, and our current study, have a few notable differences. First, we elected to evaluate a group of patients with presumed non-metastatic disease where surgical intent was curative. Including patients with known metastatic or lymph node positive disease is an extremely different cohort and the benefit of surgical intervention is dependent upon many clinical and radiographic variables as well as the ability for patients to receive adjuvant therapy $(16,17)$. Second, although we were unable to capture intraoperative surgical variables, we did not observe significant differences in outcomes with increasing severity of tumor biology relative to patient age. Finally, we elected to use 90DM as a more comprehensive endpoint that includes data on all patients who demised with surgical intervention as the primary cause of death or at least an important contributor.

Our analysis should be considered in the context of several limitations. The majority of limitations relate to data variables included in the National Cancer Database. Unfortunately, the database does not report oncologic outcomes such as disease-free, cancer specific survival or cause of death. We instead chose to focus on thirty and ninety-day mortality acknowledging that the ideal decision-making tool would be one that weighs short term morbidity and mortality risk against long term cancer survival for each individual patient. Additionally, intraoperative details are not reported in the NCDB which could have influenced our findings. Because of the lack of preoperative clinical reporting, pathologic T3b or greater disease was used as surrogate for IVC involvement of the primary tumor with the assumption that IVC involvement would impart greater potential morbidity than tumor residing only in a branch or main renal vein. The NCDB 
uses the Charlson-Deyo score to report patient comorbid conditions. This method uses a weighted calculation based on the sum of the scores of comorbid conditions, which is an inexact method of determining comorbidity. This further increases the heterogenicity of the cohort. Surgeries were completed over a 9-year period during which the use of minimally invasive approaches for surgical management of RCC was increasing. Thus, operative year may have influenced 90DM, however, this was not considered in our multivariate analysis as it was not a patient or tumor specific variable. Population databases include multiple surgeons with different levels of surgical experience which cannot be accounted for in our analysis. Certainly, single surgeon or institution cohorts may report lower 90DM; however, we believe using population data to elucidate predictors of 90DM is more appropriate due to the generalizability of these results. Finally, a split validation assessment of our nomogram is not as robust as external validation on a separate patient population; however, results would be likely be similar using another large database or institutional records due to the fact that the NCDB captures $>70 \%$ of newly diagnosed cases at over 1,500 hospitals in the United States. These findings notwithstanding our dataset is the largest to describe both 30 and 90-day mortality rates in patients presenting with localized renal masses and the first to provide a useful nomogram tool for pre-operative counseling in this setting.

We believe that our results will assist physicians in clinical-decision making and help guide patient counseling. Deciding to operate on patients presenting with suspected renal cell carcinoma is not always easy, especially in older individuals. The results of the study should not be interpreted as a suggestion to not operate on older individuals with renal masses. We advocate for a thoughtful physician-patient discussion on the competing risks of morbidity and 
mortality in this group. Trends suggest that older patients with RCC are being operated on more frequently (18). Much of this increase is due to the advent and acceptability of minimally invasive approaches with the proposed benefits of limiting complications (19). However, despite the recent surgical advances older patients have less functional reserve than younger patients and perioperative complications in this cohort are often poorly tolerated. Our preoperative tool may help to carefully select elderly patients appropriate for surgical intervention and to provide patients with appropriate counseling of the risks of perioperative mortality based on preoperative patient and tumor characteristics.

Moving forward, it is our hope to be able to confirm the external validity of our nomogram on a separate dataset. Ultimately, we plan to evaluate if these pre-operative factors are associated with disease specific and overall survival. Confirming these initial observations would aid in patient selection for surgical intervention, especially in those with advanced age, by evaluating the competing causes of morbidity and mortality. With future institutional collaboration, we hope to assess whether age, morbidity, demographics and other significant variables influence longterm results.

\section{Conclusions:}

90-day surgical mortality in patients undergoing surgery for localized non-metastatic renal cell carcinoma is independently associated with patient age, gender, stage, race and co-morbidities. Of all these factors, advanced patient age is the most predictive of surgical short-term mortality. Age appears to be more important that tumor biology and patient co-morbidities. We created a nomogram to help in pre-operative patient counseling and risk analysis prior to surgical intervention. 


\section{References:}

1. American Cancer Society: Cancer Facts and Figures 2017 [Internet]. Cancer Facts \& Figures 2017. Atlanta, Ga; [cited 2017 Aug 17]. Available from:

www.cancer.org/research/cancer-facts-statistics/all-cancer-facts-figures/cancer-factsfigures-2017.html

2. Jemal A, Simard EP, Dorell C, Noone A-M, Markowitz LE, Kohler B, et al. Annual Report to the Nation on the Status of Cancer, 1975-2009, featuring the burden and trends in human papillomavirus(HPV)-associated cancers and HPV vaccination coverage levels. J Natl Cancer Inst. 2013 Feb 6;105(3):175-201.

3. Chow W-H, Dong LM, Devesa SS. Epidemiology and risk factors for kidney cancer. Nat Rev Urol. 2010 May;7(5):245-57.

4. Chow WH, Devesa SS, Warren JL, Fraumeni JF. Rising incidence of renal cell cancer in the United States. JAMA. 1999 May 5;281(17):1628-31.

5. Hollingsworth JM, Miller DC, Daignault S, Hollenbeck BK. Rising incidence of small renal masses: a need to reassess treatment effect. J Natl Cancer Inst. 2006 Sep 20;98(18):1331-4.

6. Sun M, Thuret R, Abdollah F, Lughezzani G, Schmitges J, Tian Z, et al. Age-adjusted incidence, mortality, and survival rates of stage-specific renal cell carcinoma in North America: a trend analysis. Eur Urol. 2011 Jan;59(1):135-41.

7. Gandaglia G, Ravi P, Abdollah F, Abd-El-Barr A-E-RM, Becker A, Popa I, et al. Contemporary incidence and mortality rates of kidney cancer in the United States. Can Urol Assoc J. 2014 Jul;8(7-8):247-52.

8. Santos Arrontes D, Fernández Aceñero MJ, García González JI, Martín Muñoz M, Paniagua Andrés P. Survival analysis of clear cell renal carcinoma according to the Charlson comorbidity index. The Journal of Urology. 2008 Mar;179(3):857-61.

9. Cai M, Wei J, Zhang Z, Zhao H, Qiu Y, Fang Y, et al. Impact of age on the cancerspecific survival of patients with localized renal cell carcinoma: martingale residual and competing risks analysis. Kazembe L, editor. PLoS ONE. Public Library of Science; 2012;7(10):e48489.

10. Frank I, Blute ML, Cheville JC, Lohse CM, Weaver AL, Zincke H. An outcome prediction model for patients with clear cell renal cell carcinoma treated with radical nephrectomy based on tumor stage, size, grade and necrosis: the SSIGN score. The Journal of Urology. 2002 Dec;168(6):2395-400.

11. Fernando A, Fowler S, Van Hemelrijck M, O'Brien T, British Association of Urological Surgeons (BAUS). Who is at risk of death from nephrectomy? An analysis of thirty-day mortality after 21380 nephrectomies in 3 years of the British Association of Urological Surgeons (BAUS) National Nephrectomy Audit. BJU Int. 2017 Apr 24;120(3):358-64. 
12. Cloutier V, Capitanio U, Zini L, Perrotte P, Jeldres C, Shariat SF, et al. Thirty-day mortality after nephrectomy: clinical implications for informed consent. Eur Urol. 2009 Dec;56(6):998-1003.

13. Karakiewicz PI, Suardi N, Capitanio U, Jeldres C, Ficarra V, Cindolo L, et al. A preoperative prognostic model for patients treated with nephrectomy for renal cell carcinoma. Eur Urol. 2009 Feb;55(2):287-95.

14. Bilimoria KY, Stewart AK, Winchester DP, Ko CY. The National Cancer Data Base: a powerful initiative to improve cancer care in the United States. Ann Surg Oncol. SpringerVerlag; 2008 Mar;15(3):683-90.

15. Boffa DJ, Rosen JE, Mallin K, Loomis A, Gay G, Palis B, et al. Using the National Cancer Database for Outcomes Research: A Review. JAMA Oncol. 2017 Feb 23.

16. Flanigan RC, Mickisch G, Sylvester R, Tangen C, Van Poppel H, Crawford ED. Cytoreductive nephrectomy in patients with metastatic renal cancer: a combined analysis. The Journal of Urology. 2004 Mar;171(3):1071-6.

17. Hanna N, Sun M, Meyer CP, Nguyen PL, Pal SK, Chang SL, et al. Survival Analyses of Patients With Metastatic Renal Cancer Treated With Targeted Therapy With or Without Cytoreductive Nephrectomy: A National Cancer Data Base Study. J Clin Oncol. 2016 Sep 20;34(27):3267-75.

18. Kim SP, Gross CP, Meropol N, Kutikov A, Smaldone MC, Shah ND, et al. National treatment trends among older patients with T1-localized renal cell carcinoma. Urol Oncol. 2017 Mar;35(3):113.e15-113.e21.

19. Guzzo TJ, Allaf ME, Pierorazio PM, Miller D, McNeil BK, Kavoussi LR, et al. Perioperative outcomes of elderly patients undergoing laparoscopic renal procedures. Urology. 2009 Mar;73(3):572-6. 